\title{
Ketamine: A Neuroanesthesiologist's Friend or Foe?
}

\author{
Ankur Luthra $\quad$ Girija Prasad Rath² \\ ${ }^{1}$ Department of Anaesthesia and Intensive Care, Postgraduate \\ Institute of Medical Education and Research, Chandigarh, India \\ ${ }^{2}$ Department of Neuroanaesthesiology and Critical Care, \\ All India Institute of Medical Sciences, New Delhi, India \\ J Neuroanaesthesiol Crit Care 2018;5:77-82.
}

\begin{abstract}
Address for correspondence Ankur Luthra, MD, DM, H. No. 979, Aashirwad Enclave, Sector 49-A, Chandigarh 160047, India

(e-mail: zazzydude979@gmail.com).
\end{abstract}

\begin{abstract}
Keywords

- $N$-methyl-D-aspartate antagonist

- excitotoxicity

- neuroprotection

- neuroinflammation

Ketamine, an N-methyl-D-aspartate (NMDA) receptor antagonist, introduced into clinical practice six decades ago, has often been the worst fear of a neuroanesthesiologist due to its concerns of causing a rise in intracranial pressure and increasing cerebral blood volume. However, the recent literature clarifies that it may have a beneficial role, even in neurosurgical patients, because of its propensity to cause neuroprotection through antiglutamatergic action on the NMDA receptors in the ischemic brain. Apart from having an anticonvulsive role in refractory status epilepticus, its inherent property of increasing blood flow to the ischemic areas offers protection to the penumbral zone, thus preventing secondary brain injuries. Also, it has been widely used as an analgesic at subanesthetic doses post spine surgeries. Recently, enough scientific evidence has been published in favor of ketamine establishing the fact that it does not cause a rise in intracranial pressure when the patient is mechanically ventilated and normocarbia is maintained. It has also been used during evoked potential monitoring as it amplifies signals even under general anesthesia and proves to be quite effective during neuromonitoring. However, its adverse effects of increasing muscle tone, excessive salivation, emergence delirium, agitation, and "out of body" experiences with long-term psychomimetic effects and potential to cause addiction have precluded its widespread use.

This review on ketamine summarizes the benefits of using it in neurosurgical anesthesia with the aim of removing the fears we had in the past, which did not seem to be evidence based.
\end{abstract}

\section{Introduction}

The journey of "ketamine" dates back to the 1950s when scientists at Park Davis Laboratories, Detroit, Michigan, formulated phencyclidine (PCP), trials of which were conducted on humans in 1958 under the name of Sernyl. With growing clinical evidence, it became clear that PCP was not suitable for human anesthesia. One of its derivatives, synthesized in 1962 by Calvin Stevens, led to excellent short-acting anesthesia. It was selected for clinical trials in humans as CI-581 [2- (O-chloro-phenyl)-2-methylamino cyclohexanone]. ${ }^{1}$ As it was a ketone together with an amine, it was named "ketamine." The commercially available ketamine is a racemic mixture of two enantiomers $\mathrm{R}(-)$ ketamine and
S (+) ketamine. $S(+)$ isomer has four times greater affinity for the NMDA receptors than $\mathrm{R}(-)$ isomer, and thus it has a greater anesthetic and analgesic potency.

The burden of ischemic injury to the brain, for example, in cerebrovascular accident (CVA), traumatic brain injury (TBI), tumors, hemorrhage, etc. is ever increasing, many of which requiring a neurosurgery. Continued advancements in anesthetic treatments during the perioperative period may be one of the significant ways to improve the overall patient outcomes. An ideal neuroanesthetic agent should possess neuroprotective properties such as reducing cerebral metabolic rate $\left(\mathrm{CMRO}_{2}\right)$ and cerebral blood volume $(\mathrm{CBV})$, preventing increase in intracranial pressure (ICP),

\section{received}

February 22, 2018

accepted

April 16, 2018

published online

May 24, 2018
DOI https://doi.org/

10.1055/s-0038-1654746.

ISSN 2348-0548.
Copyright @2018 Indian Society of Neuroanaesthesiology and Critical Care
License terms

(a) (1) $\ominus \circledast$ 
maintaining cerebral autoregulation and $\mathrm{CO}_{2}$ reactivity, preventing seizure activity. Also, it should neither be a vasodilator or a myocardial depressant nor an adrenal suppressant. However, till date, an ideal neuroanesthetic agent is yet to be available in clinical practice.

\section{Mechanism of Action: Neuropharmacology}

Ketamine is an intravenously administered general anesthetic agent that primarily acts through noncompetitive binding to the $N$-methyl-D-aspartate (NMDA) receptor. ${ }^{2}$ The secondary mechanisms of action include those at the $\mu$ opioid, muscarinic and monoaminergic receptors, voltage-gated sodium, and the L-type calcium channels. ${ }^{3-6}$ It is also known to inhibit interleukin-6 (IL-6) and catecholamine reuptake. ${ }^{7}$ These mechanisms clinically lead to induction of a dissociative cataleptic state and also provide analgesia, hypnosis, bronchodilation, and sympathetic stimulation. ${ }^{8}$ This is clinically advantageous as administering ketamine with a $\gamma$-aminobutyric acid (GABA) agonist reduces its sympathomimetic effect that results in a stable hemodynamic state and reduces the patient's requirement for vasoactive medications. ${ }^{9}$

The traditional pharmacologic neuroprotection with neuroanesthetics is achieved by increasing oxygen delivery and decreasing the pathologic mechanisms that result in neuronal cell death. ${ }^{10}$ Glutamate excitotoxicity is the principal mechanism of neuronal cell death, and unlike other anesthetic agents, ketamine possesses a unique pharmacologic property of antagonizing this excitotoxicity via NMDA receptor upregulation during cerebral ischaemia. ${ }^{11}$ However, ketamine has not been so widely used in neurosurgical anesthesia practice due to the concerns of increasing $\mathrm{CBV}$, which contributes to rise in ICP. Earlier studies noted a rise in ICP with the use of ketamine for sedation in spontaneously breathing patients that led to hypercarbia, and hence cerebral vasodilation. ${ }^{12}$ Recent literature and reevaluation of this subject have paved the way of how this drug should be looked at and made us rethink the paradigm, thus revisiting its role in neuroanesthesia practice. It has now been clarified that using ketamine as an adjuvant to other neuroanesthetic agents with maintenance of normocapnia through mechanical ventilation does not lead to increase in ICP. ${ }^{13-16}$

Another beneficial effect of ketamine in neurosurgical patients is through occurrence of both neuroprotective properties of physiological synaptic NMDA receptor activation and neurodestruction caused by excitotoxic extrasynaptic NMDA receptor activation. Under physiologic states, ketamine is known to oversuppress the physiologic NMDA receptor activation preventing the neuroprotective cascade. ${ }^{17}$ However, when administered during cerebral ischemia, it antagonizes the glutamate activation of the extrasynaptic NMDA receptor activation, thus preventing the neurodestructive cascade and reducing neuronal cell death.

\section{Mechanism of Neuroprotection}

There are different neuroprotective and neurodestructive pathways mediated through synaptic and extrasynaptic NMDA receptor activation. Under physiologic conditions, the beneficial effects are mediated through phosphoinositide-3-kinase
(PI3K)-AKT pathway that inhibits proapoptotic glycogen synthase kinase $3 \beta$ (GSK3 $\beta$ ), forkhead box 0 (FOXO), and p53 transcription factor genes. ${ }^{18-21}$

The synaptic stimulation of NMDA receptor also activates striatal-enriched tyrosine phosphatase (STEP) degradation along with extracellular signal-regulated kinase $1 / 2\left(\right.$ ERK $\left.^{1} / 2\right)$ activation, leading to cell survival. ${ }^{22}$ Also, the associated $\mathrm{Ca}^{2+}$ influx stimulates CREB (cyclic adenosine monophosphate [cAMP] response element-binding protein) that increases tolerance of neurons to ischemic events, thus delaying apoptosis and in turn also explaining neuronal ischemic preconditioning. ${ }^{19,23}$ All these pathways are triggered by synaptic NMDA receptor activation, which may be oversuppressed by ketamine leading to neurodestruction under physiologic conditions.

However, under ischemic conditions, use of ketamine is advantageous. Ischemia induces excitotoxic process involving presynaptic release of large amounts of glutamate, which stimulates extrasynaptic NMDA receptors. ${ }^{23}$ These higher levels of glutamate then overactivate postsynaptic NMDA receptor protein kinase $C$ and tyrosine kinase signaling cascades..$^{24,25}$ They phosphorylate the NMDA receptors and upregulate the signaling currents, thus amounting to increase in NMDA receptor activation. Whenever there is increase in excitotoxic glutamate release, it is the extrasynaptic NMDA receptors that mediate neurodestruction and override neuroprotective pathways. ${ }^{23,25}$ Extrasynaptic NMDA receptor activation induces neuronal injury by causing excessive influx of $\mathrm{Ca}^{2+}$, which, in turn, activates calpain, disabling the $\mathrm{Na}^{+} / \mathrm{Ca}^{2+}$ exchanger and decreases $\mathrm{Ca}^{2+}$ efflux, further augmenting apoptosis. ${ }^{26} \mathrm{This} \mathrm{Ca}^{2+}$ also activates neuronal nitric oxide synthase leading to overproduction of nitric oxide (NO) and damaging the mitochondria. In addition, calpain breaks down STEP, activates p38, and promotes nuclear import of FOXO. ${ }^{27}$ These pathways ultimately culminate in cell death by apoptosis or necrosis.

\section{Review of Clinical Evidence}

Ketamine has been under extensive clinical research since its introduction in the 1960s. Earlier it was discouraged in neurosurgical patients due to the concerns of raised ICP. However, ample clinical evidence now supports its use even in patients with neurosurgical pathologies as it does not have any deleterious cerebral hemodynamic or neurologic consequences.

\section{Traumatic Brain Injury}

Bar-Joseph et al ${ }^{13}$ studied neurologic effects of ketamine prospectively in intubated children with raised ICP who were sedated with midazolam, 2 to $5 \mu \mathrm{g} / \mathrm{kg} / \mathrm{min}$, and morphine, 20 to $50 \mu \mathrm{g} / \mathrm{kg} / \mathrm{min}$. They were administered ketamine boluses $(1-1.5 \mathrm{mg} / \mathrm{kg}$ ) during episodes of ICP elevation and before any sort of stimulating events. The authors observed that ketamine decreased the ICP by an average of $30 \%(p<0.001)$ and achieved modest increase in cerebral perfusion pressure (CPP) $(p<0.005)$. This study concluded that, for neurosurgical sedation, adjuvant administration of ketamine with other anesthetic agents resulted in favorable cerebral hemodynamic responses. 
Bourgoin et al ${ }^{14}$ conducted a randomized, double-blinded, trial comparing patients with TBI in the intensive care unit (ICU) who received sedation with either ketamine/midazolam or sufentanil/midazolam, and they observed no statistical difference in ICP between the two groups $(p=0.28)$. Six months postinjury, the Glasgow outcome score (GOS) of each group was measured to determine neurologic function of each patient, and no statistical difference could be discerned $(p=0.99)$. Similarly, a retrospective study was conducted by Grathwohl et $\mathrm{al}^{28}$ who compared the neurologic outcome of 252 patients with TBI receiving total intravenous anesthesia (TIVA) with propofol and ketamine to TIVA without ketamine and to volatile anesthesia without ketamine. They concluded that there was no statistically significant difference in the number of patient deaths between the groups $(p=0.36)$ and no statistical difference in the GOS of each group $(p=0.47)$. Both these studies illustrated that as an adjuvant, ketamine did not worsen patient outcomes and was statistically indifferent from the outcomes of patients who received anesthesia with volatile anesthetics, propofol, opioid, or benzodiazepines.

Långsjö et $\mathrm{al}^{12}$ studied that ketamine led to an increase in regional cerebral blood flow in the anterior cingulate and frontal cortices and observed that $\mathrm{CMRO}_{2}$ did not directly correlate with cerebral blood flow changes. It did not increase after ketamine was administered. However, this study was performed in spontaneously breathing individuals, and the results cannot be extrapolated to neurosurgical patients who usually are under controlled mechanical ventilation with maintenance of normocarbia.

In a meta-analysis published by Cohen et a ${ }^{29}$ regarding the effects of ketamine on intracranial and cerebral perfusion pressure and health outcomes that included 10 studies and 953 critically ill patients. Two studies reported a slight decrease in ICP within 10 minutes of ketamine administration, and two studies reported an increase. However, none of the studies reported significant differences in CPP, neurologic outcomes, ICU length of stay, and mortality. Another recent meta-analysis published by Zeiler et a ${ }^{30}$ included studies of 101 adult and 55 pediatric patients and stated that ICP did not increase in any of these studies during ketamine administration. Rather, three studies reported a significant decrease in ICP with ketamine bolus. There currently exists Oxford level $2 \mathrm{~b}$, grade $\mathrm{C}$ evidence to support that ketamine does not increase ICP in severe TBI patients who are sedated and ventilated, and in fact, may lower it in selected cases.

In TBI, the contused segment of the brain forms the core and is usually surrounded by a penumbra of "at risk" tissue that may suffer secondary brain injury. Use of ketamine that causes an increase in mean arterial pressure and possibly an increase in CPP may lead to increased cerebral blood flow to these "at risk" and vulnerable areas, thereby preventing secondary ischemic injury.

Ketamine, when used as an induction agent for rapid sequence intubation in undifferentiated critically ill patients or patients with major trauma where neurologic injury has not been ruled out, still remains an inconclusive debate for emergency physicians. A very essential role of the emergency care provider managing such a patient is to ensure minimal secondary insults to the brain during the resuscitation phases of the patient's care. These patients might be irritable, in pain, and combative, and hence prone to a high systemic sympathetic response, and thus already raised ICP. Thiopentone, propofol, or fentanyl might lead to significant hypotension during induction, particularly in hypovolemic patients, which might worsen outcome of such patients.

Various studies have established a strong association between the degree and duration of hypotension (a systolic blood pressure of $<90 \mathrm{~mm} \mathrm{Hg}$ in adults) and worse neurologic outcomes in patients with TBI. Hence, it is imperative to avoid using induction agents such as barbiturates, opioids, and benzodiazepines that cause or may exacerbate any preexisting hemodynamic instability and may worsen the hypotension and cause further cerebral ischemia. Ketamine, having the advantage of maintaining a neutral hemodynamic profile, may be safely used. ${ }^{31}$ Etomidate may have an edge over other induction agents in this regard. However, in light of the uncertainty over the clinical significance of the adrenal suppression associated with it and the frequent occurrence of sepsis in patients with major trauma, the available evidence suggests that ketamine should be considered as an alternative induction agent or as an adjunct to other intravenous (IV) agents. ${ }^{32}$ The current practice of avoiding ketamine in rapid sequence induction and intubation of head-injured patients, for the fear of raising ICP, is not evidence based. Further prospective, randomized studies need to be done before refuting the use of ketamine in emergency settings.

\section{Delayed Cerebral Ischemia}

Another major area of focus for evaluation of uses of ketamine in neurosurgical population is delayed cerebral ischemia (DCI). DCI is a clinical syndrome of focal neurologic and cognitive deficits that occurs in approximately 30\% of patients 3 to 14 days after the initial subarachnoid hemorrhage (SAH). ${ }^{33}$ While aneurysmal rebleeding is another significant complication in the initial few hours following the bleed, DCI remains one of the most important causes of morbidity and mortality in patients who survive to definitive aneurysm treatment. ${ }^{34}$ Previously, it was widely established that the primary mechanism leading to delayed neurologic deterioration after SAH was vasospasm or narrowing of caliber of cerebral blood vessels (due to endothelial hypertrophy and vasoconstriction), leading to brain tissue ischemia that was mediated by extravasated blood in the subarachnoid space. However, lately, research studies have proved that looking beyond vasospasm is essential and that a multifactorial etiology has been put forward. Among those factors, cortical spreading depression plays a major role. The term describes a depolarization wave in the cerebral cortex, particularly in the gray matter that propagates at 2 to $5 \mathrm{~mm} / \mathrm{min}$ across the brain and results in depression of evoked and spontaneous electroencephalographic (EEG) activity. ${ }^{35}$ This propagation of the cortical spreading depression (CSD) wave silences spontaneous and evoked synaptic activity in the brain for 5 to 15 minutes and is followed by spontaneous return to normal function. It is present in the normal brain also. However, in 
the injured brain, the return to normal function is prolonged, which contributes to neuronal ischemia and microvascular dysfunction. With each wave of cortical spreading depression, the under-perfused segments of the brain that were becoming increasingly ischemic do not recover. It has been postulated to be one of the mechanisms in TBI and in migraine with aura, with less severity though. Few animal studies have demonstrated the role of ketamine in suppressing this cortical spreading depolarization.

A small randomized prospective multiple crossover trial was conducted by Carlson et al, ${ }^{36}$ who studied 10 patients with either SAH or TBI. A $1 \times 6$ electrocorticographic (ECoG) strip was placed on the brain surface at the time of craniotomy, and patients were then placed on a randomized alternating 6-hour dosing schedule of ketamine. It was observed that ketamine effectively inhibits cortical spreading depolarization after acute neurologic insult in a dose-dependent fashion (a dose of $>1.15 \mathrm{mg} / \mathrm{kg} / \mathrm{h}$ was required to inhibit the cortical spreading depolarization effectively). However, whether it really contributes to a clinical benefit is not yet known. Further prospective studies will be required to conclusively define its advantage in SAH and TBI patients.

\section{Ischemic Stroke}

Another potential area that still needs to be researched is the prevention of increase in infarct size or volume postischemic stroke in a vast majority of patients to prevent major morbidity and mortality. An early preclinical evidence on use of ketamine during sedation in mice has been found to reduce infarct size post induction of ischemic stroke and its treatment by recombinant tissue plasminogen activator for thrombolysis by Gakuba et al. ${ }^{37}$ Based on this study, recently KETA (Ketamine for Thrombolysis in Acute Ischemic Stroke) trial that is a double-blinded, randomized, controlled pilot trial aiming to determine whether co-administration of ketamine with recombinant plasminogen activator (rtPA) for thrombolysis in acute ischemic stroke compared with rtPA co-administered with placebo decreases cerebral infarction growth in diffusion-weighted imaging between admission and day 1 has been initiated. Eligibility applies to 50 patients with symptomatic ischemic stroke seen within 4.5 hours of onset with occlusion of the middle cerebral artery (MCA) or distal internal carotid artery (ICA), and no contraindication to IV tPA-mediated thrombolysis and eligible to endovascular treatment of stroke (i.e., thrombectomy). The dose of ketamine that is being investigated is $0.15 \mathrm{mg} / \mathrm{kg}$ IV (maximum $15 \mathrm{mg}$ ) followed by an infusion of $0.15 \mathrm{mg} / \mathrm{kg}$ over $60 \mathrm{~min}-$ utes (maximum $15 \mathrm{mg}$ ).

\section{Analgesia and Neuroinflammation}

Ketamine has also been long known for its excellent analgesia at subanesthetic doses of $0.2 \mathrm{mg} / \mathrm{kg}$, and this property has also been well utilized in neurosurgical group of patients particularly post spine surgery as well as an adjuvant to local anesthetics for infiltration to prevent post craniotomy pain. ${ }^{38}$ A recent meta-analysis was published by Pendi et al, ${ }^{39}$ which included 14 randomized controlled trials (RCTs) comprising 649 patients who were administered adjunctive ketamine to reduce postoperative analgesic requirements of opioids post spine surgery. The ketamine group had less cumulative morphine equivalent consumption at $4,8,12$, and 24 hours following spine surgery $(p<0.05)$ and lower postoperative pain scores at 6,12 , and 24 hours $(p<0.05)$ without any adverse events or harmful effects. Preventing central sensitization in the dorsal horn neurons (interfere with pain transmission in spinal cord), acting as $\mu$ receptor agonist (primarily by S-enantiomer), and inhibiting nitric oxide synthase are some of the proposed mechanisms of pain control by ketamine. It also inhibits IL-6, tumor necrosis factor $\alpha$ and IL-8 production, and other proinflammatory cytokine activity in both central and peripheral nervous systems. It also prevents neuroinflammation by inhibiting these cytokines in glial cells and microglia of the central nervous system. This also has been proposed to be one of the mechanisms of neuroprotection by ketamine. Because of its ability to inhibit neuroinflammation, it has also shown to attenuate postoperative cognitive dysfunction particularly in the elderly after cardiac surgery ${ }^{40}$ and orthopaedic surgery. ${ }^{41}$ However, this effect has not widely been extrapolated to postoperative cognitive dysfunction occurring after most neurosurgical procedures. The reasons, however, remain unclear and suggest that other mechanisms apart from neuroinflammation might play a role in postoperative cognitive dysfunction post neurosurgery.

\section{Epilepsy Surgery and Refractory Status Epilepticus}

Ketamine has demonstrated both pro- and anticonvulsant properties depend on the dosage and chronicity of the convulsive activity. In refractory and super-refractory status epilepticus, ketamine has been used post 48 hours of seizure activity with varying degrees of success. A dosage of 2 to $5 \mathrm{mg} / \mathrm{kg}$ to attenuate seizures has been safely used in both adult and pediatric population. ${ }^{42,43}$ During prolonged seizures, there is downregulation of GABA receptors whose activities gradually decrease; thus, the commonly used firstand second-line antiepileptic drugs gradually fail. Simultaneously, there is upregulation of activities of glutamatergic NMDA receptors, often causing refractory status epilepticus and thus providing the possibility of the use of ketamine to treat refractory status. ${ }^{43}$ However, ketamine possesses proconvulsant properties also and is known to activate epileptic foci, thereby having a potential role during ECoG monitoring. Ketamine significantly caused more ECoG identifiable seizures than methohexital or thiopentone and even facilitated extra-temporal seizure focus localization in comparison with temporal focus $(p<0.05){ }^{44}$ These ECoG seizures provoked after administration of ketamine improved the localization of the area to be resected during epilepsy surgery, more so in extratemporal epileptic foci. ${ }^{45}$

\section{Evoked Potentials}

A vital part of all neurosurgical procedures is neuromonitoring, and one of the essential intraoperative neuromonitors is evoked potential. Unfortunately, many of the general anesthetic agents including volatile anesthetics, benzodiazepines, and high-dose barbiturates suppress the amplitude of evoked potentials and interfere with neuromonitoring. However, 
ketamine has been found in several preclinical and clinical studies to actually increase evoked potential amplitude, and thus it may be used as an analgesic adjunct in procedures in which evoked potential monitoring is critical. It can increase the amplitude of the cortical somatosensory evoked potential (SSEP) and motor evoked potential (MEP) in muscle and spinal recorded tracings after spinal stimulation. ${ }^{45,46}$ This effect may be mediated by the same mechanisms that potentiate the H-reflex. However, effects on subcortical and peripheral SSEPs are minimal, and so are effects on myogenic MEPs. ${ }^{47}$

\section{Analgosedation Regimens}

Recent animal and human studies have suggested that ketamine does not alter cerebral autoregulation, ${ }^{48,49}$ and when compared with opioids such as sufentanil, it has not demonstrated any elevation in ICP. ${ }^{50}$ In fact, ketamine in a dose range of 1.5 to $3 \mathrm{mg} / \mathrm{kg}$ in combination with propofol has been shown to reduce ICP in patients with TBI with no significant difference in CPP, jugular venous oxygenation, and MCA flow, with induction of low-amplitude fast activity EEG, with marked depression such as burst suppression..$^{51}$

Ketamine has also demonstrated to facilitate routine bedside procedures such as endotracheal suctioning. In a study by Caricato et al, ${ }^{52}$ racemic ketamine $(100 \mu \mathrm{g} / \mathrm{kg} / \mathrm{min}$ for 10 minutes) used before endotracheal suctioning was not associated with significant variations in СРP and jugular venous oxygenation although ketamine was not effective in controlling ICP elevation during this time completely. Ketamine has often been used in conjunction with midazolam or propofol, ${ }^{14}$ and the concurrent use results in less requirements of vasopressors. ${ }^{9}$ It is also well suited for analgosedation after major spine surgeries with an added opioid sparing effect.

Oral ketamine has been successfully used to treat central neuropathic pain as well as post stroke and thalamic pain, which were refractory to conventional pain treatments in recently published case reports. ${ }^{53,54}$ However, the side effects of ketamine such as dysphoria, hallucinations, and paranoid feelings were attenuated by the concurrent use of benzodiazepines.

\section{Conclusion}

A variety of uses of ketamine in general anesthesia in the settings of treating chronic pain, active bronchospasm or severe asthma, burn dressings, congenital heart diseases (particularly tetralogy of Fallot and other right to left shunts), prehospital and in battlefields, as premedication to infants and children, and in trauma and hypotensive patients have been elaborated elsewhere in the literature and are beyond the scope of this article. However, its adverse effects of increasing muscle tone, excessive salivation, emergence delirium, agitation, and "out of body" experiences with long-term psychomimetic effects and potential to cause addiction have precluded its generalized use. It is now being researched as a neuroprotectant in patients with TBI, delayed cerebral ischemia, stroke, and epilepsy. It is being extensively used as an excellent analgesic in spine surgery patients and has proved its promising role in providing excellent analgosedation during ICU sedation in patients with head injury. Its role in suppressing refractory status epilepticus is well established, and recently growing evidence favors its role in augmenting evoked potentials intraoperatively, as well as provoking seizures during electroconvulsive therapy for refractory depression.

In neurosurgical population, however, a dilemma still continues over its use for the fear or myths of raising ICP under certain conditions. But, looking at the various advantages and benefits in neurosurgery and neurointensive care, it is time to rethink the paradigm and evaluate it as an adjuvant to other IV anesthetic agents and should be more frequently used in the armamentarium of a neuroanesthetist or an intensivist caring for neurosurgical patients in both operating rooms and ICUs.

\section{Conflict of Interest}

None.

\section{References}

1 Domino EF. Taming the ketamine tiger. 1965. Anesthesiology 2010;113(3):678-684

2 Salt TE, Wilson DG, Prasad SK. Antagonism of $N$-methylaspartate and synaptic responses of neurones in the rat ventrobasal thalamus by ketamine and MK-801. Br J Pharmacol 1988;94(2):443-448

3 Durieux ME. Inhibition by ketamine of muscarinic acetylcholine receptor function. Anesth Analg 1995;81(1):57-62

4 Smith DJ, Bouchal RL, deSanctis CA, et al. Properties of the interaction between ketamine and opiate binding sites in vivo and in vitro. Neuropharmacology 1987;26(9):1253-1260

5 Crisp T, Perrotti JM, Smith DL, Stafinsky JL, Smith DJ. The local monoaminergic dependency of spinal ketamine. Eur J Pharmacol 1991;194(2-3):167-172

6 Baum VC, Tecson ME. Ketamine inhibits transsarcolemmal calcium entry in guinea pig myocardium: direct evidence by single cell voltage clamp. Anesth Analg 1991;73(6): 804-807

7 Lundy PM, Lockwood PA, Thompson G, Frew R. Differential effects of ketamine isomers on neuronal and extraneuronal catecholamine uptake mechanisms. Anesthesiology 1986;64(3):359-363

8 Hirota K, Lambert DG. Ketamine: its mechanism(s) of action and unusual clinical uses. Br J Anaesth 1996;77(4):441-444

9 Kolenda H, Gremmelt A, Rading S, Braun U, Markakis E. Ketamine for analgosedative therapy in intensive care treatment of head-injured patients. Acta Neurochir (Wien) 1996;138(10):1193-1199

10 Urwin SC, Menon DK. Comparative tolerability of sedative agents in head-injured adults. Drug Saf 2004;27(2):107-133

11 Hudetz JA, Pagel PS. Neuroprotection by ketamine: a review of the experimental and clinical evidence. J Cardiothorac Vasc Anesth 2010;24(1):131-142

12 Långsjö JW, Kaisti KK, Aalto S, et al. Effects of subanesthetic doses of ketamine on regional cerebral blood flow, oxygen consumption, and blood volume in humans. Anesthesiology 2003;99(3):614-623

13 Bar-Joseph G, Guilburd Y, Tamir A, Guilburd JN. Effectiveness of ketamine in decreasing intracranial pressure in children with intracranial hypertension. J Neurosurg Pediatr 2009;4(1):40-46

14 Bourgoin A, Albanèse J, Wereszczynski N, Charbit M, Vialet $\mathrm{R}$, Martin C. Safety of sedation with ketamine in severe head injury patients: comparison with sufentanil. Crit Care Med 2003;31(3):711-717 
15 Nagase K, Iida H, Ohata H, Dohi S. Ketamine, not propofol, attenuates cerebrovascular response to carbon dioxide in humans with isoflurane anesthesia. J Clin Anesth 2001;13(8):551-555

16 Sakai K, Cho S, Fukusaki M, Shibata O, Sumikawa K. The effects of propofol with and without ketamine on human cerebral blood flow velocity and $\mathrm{CO}(2)$ response. Anesth Analg 2000;90(2):377-382

17 Hardingham GE. Coupling of the NMDA receptor to neuroprotective and neurodestructive events. Biochem Soc Trans 2009;37(Pt 6):1147-1160

18 Lafon-Cazal M, Perez V, Bockaert J, Marin P. Akt mediates the anti-apoptotic effect of NMDA but not that induced by potassium depolarization in cultured cerebellar granule cells. Eur J Neurosci 2002;16(4):575-583

19 Soriano FX, Papadia S, Hofmann F, Hardingham NR, Bading $\mathrm{H}$, Hardingham GE. Preconditioning doses of NMDA promote neuroprotection by enhancing neuronal excitability. J Neurosci 2006;26(17):4509-4518

20 Lau D, Bading H. Synaptic activity-mediated suppression of p53 and induction of nuclear calcium-regulated neuroprotective genes promote survival through inhibition of mitochondrial permeability transition. J Neurosci 2009;29(14):4420-4429

21 Al-Mubarak B, Soriano FX, Hardingham GE. Synaptic NMDAR activity suppresses FOXO1 expression via a cis-acting FOXO binding site: FOXO1 is a FOXO target gene. Channels (Austin) 2009;3(4):233-238

22 Xu J, Kurup P, Zhang Y, et al. Extrasynaptic NMDA receptors couple preferentially to excitotoxicity via calpain-mediated cleavage of STEP. J Neurosci 2009;29(29):9330-9343

23 Hardingham GE, Fukunaga Y, Bading H. Extrasynaptic NMDARs oppose synaptic NMDARs by triggering CREB shut-off and cell death pathways. Nat Neurosci 2002;5(5):405-414

24 Liu Y, Zhang G, Gao C, Hou X. NMDA receptor activation results in tyrosine phosphorylation of NMDA receptor subunit 2A(NR2A) and interaction of Pyk2 and Src with NR2A after transient cerebral ischemia and reperfusion. Brain Res 2001;909(1)(2):51-58

25 Lan JY, Skeberdis VA, Jover T, et al. Protein kinase C modulates NMDA receptor trafficking and gating. Nat Neurosci 2001;4(4):382-390

26 Ankarcrona M, DypbuktJM, Bonfoco E, et al. Glutamate-induced neuronal death: a succession of necrosis or apoptosis depending on mitochondrial function. Neuron 1995;15(4):961-973

27 Dick O, Bading H. Synaptic activity and nuclear calcium signaling protect hippocampal neurons from death signal-associated nuclear translocation of FoxO3a induced by extrasynaptic $N$-methyl-D-aspartate receptors. J Biol Chem 2010;285(25):19354-19361

28 Grathwohl KW, Black IH, Spinella PC, et al. Total intravenous anesthesia including ketamine versus volatile gas anesthesia for combat-related operative traumatic brain injury. Anesthesiology 2008;109(1):44-53

29 Cohen L, Athaide V, Wickham ME, Doyle-Waters MM, Rose NGW, Hohl CM. The effect of ketamine on intracranial and cerebral perfusion pressure and health outcomes: a systematic review. Ann Emerg Med 2015;65(1):43-51.e2

30 Zeiler FA, Teitelbaum J, West M, Gillman LM. The ketamine effect on ICP in traumatic brain injury. Neurocrit Care 2014;21(1):163-173

31 Bucher J, Koyfman A. Intubation of the neurologically injured patient. J Emerg Med 2015;49(6):920-927

32 Jabre P, Combes X, Lapostolle F, et al; KETASED Collaborative Study Group. Etomidate versus ketamine for rapid sequence intubation in acutely ill patients: a multicentre randomised controlled trial. Lancet 2009;374(9686):293-300

33 Dorsch NW, King MT. A review of cerebral vasospasm in aneurysmal subarachnoid haemorrhage. Part I: Incidence and effects. J Clin Neurosci 1994;1(1):19-26
34 Kassell NF, Torner JC, Haley EC Jr, Jane JA, Adams HP, Kongable GL. The International Cooperative Study on the Timing of Aneurysm Surgery. Part 1: overall management results. J Neurosurg 1990;73(1):18-36

35 Leao A. Spreading depression of activity in the cerebral cortex. J Neurophysiol 1944;7:359

36 Carlson AP, Abbas M, Alunday R, Qeadank F, Shuttleworth CW. 375 ketamine inhibits cortical spreading depolarization in acute brain injury: a prospective randomized multiple crossover trial. Neurosurgery 2017;64 (e1):288-289

37 Gakuba C, Gauberti M, Mazighi M, Defer G, Hanouz JL, Vivien D. Preclinical evidence toward the use of ketamine for recombinant tissue-type plasminogen activator-mediated thrombolysis under anesthesia or sedation. Stroke 2011;42(10):2947-2949

38 Nemergut EC, Durieux ME, Missaghi NB, Himmelseher S. Pain management after craniotomy. Best Pract Res Clin Anaesthesiol 2007;21(4):557-573

39 Pendi A, Field R, Farhan SD, Eichler M, Bederman SS. Perioperative ketamine for analgesia in spine surgery: a meta-analysis of randomized controlled trials. Spine 2018;43(5):E299-E307

40 Hudetz JA, Iqbal Z, Gandhi SD, et al. Ketamine attenuates post-operative cognitive dysfunction after cardiac surgery. Acta Anaesthesiol Scand 2009;53(7):864-872

41 Lee KH, Kim JY, Kim JW, Park JS, Lee KW, Jeon SY. Influence of ketamine on early postoperative cognitive function after orthopedic surgery in elderly patients. Anesth Pain Med 2015;5(5):e28844

42 Synowiec AS, Singh DS, Yenugadhati V, Valeriano JP, Schramke CJ, Kelly KM. Ketamine use in the treatment of refractory status epilepticus. Epilepsy Res 2013;105(1)(2):183-188

43 Fang Y, Wang X. Ketamine for the treatment of refractory status epilepticus. Seizure 2015;30:14-20

44 Rysz A, Bachański M, Bidziński J, Bacia T. [The comparison of ketamine with methohexital and thiopental in the intraoperative EEG in drug-resistant epilepsy] [in Polish] Neurol Neurochir Pol 1998;32(2, Suppl 2):237-245

45 Kano T, Shimoji K. The effects of ketamine and neuroleptanalgesia on the evoked electrospinogram and electromyogram in man. Anesthesiology 1974;40(3):241-246

46 Schubert A, Licina MG, Lineberry PJ. The effect of ketamine on human somatosensory evoked potentials and its modification by nitrous oxide. Anesthesiology 1990;72(1):33-39

47 Glassman SD, Shields CB, Linden RD, Zhang YP, Nixon AR, Johnson JR. Anesthetic effects on motor evoked potentials in dogs. Spine 1993;18(8):1083-1089

48 Schmidt A, Ryding E, Akeson J. Racemic ketamine does not abolish cerebrovascular autoregulation in the pig. Acta Anaesthesiol Scand 2003;47(5):569-575

49 Engelhard K, Werner C, Möllenberg O, Kochs E. S(+)-ketamine/ propofol maintain dynamic cerebrovascular autoregulation in humans. Can J Anaesth 2001;48(10):1034-1039

50 Loflin R, Koyfman A. When used for sedation, does ketamine increase intracranial pressure more than fentanyl or sufentanil? Ann Emerg Med 2015;65(1):55-56

51 Albanèse J, Arnaud S, Rey M, Thomachot L, Alliez B, Martin C. Ketamine decreases intracranial pressure and electroencephalographic activity in traumatic brain injury patients during propofol sedation. Anesthesiology 1997;87(6):1328-1334

52 Caricato A, Tersali A, Pitoni S, et al. Racemic ketamine in adult head injury patients: use in endotracheal suctioning. Crit Care 2013;17(6):R267

53 Vick PG, Lamer TJ. Treatment of central post-stroke pain with oral ketamine. Pain 2001;92(1)(2):311-313

54 O’Brien SL, Pangarkar S, Prager J. The use of ketamine in neuropathic pain. Curr Phys Med Rehabil Rep 2014;2:128-145 